\title{
Intelligent Tutoring Systems: Confluence of Information Science and Cognitive Science
}

\author{
A. Patel \\ De Montfort University, U.K. \\ apatel@dmu.ac.uk \\ D. Russell \\ De Montfort University, U.K \\ drussell@dmuac.uk
}

\author{
Kinshuk \\ Massey University, New Zealand \\ kinshuk@massey.ac.nz \\ R. Oppermann \\ GMD, Germany \\ oppermann@gmd.de
}

\begin{abstract}
The advent of Internet as a global communication medium has brought a new focus on an area of research in designing Intelligent Tutoring System (ITS) that has not been adequately considered so far. In the main, this has been due to the localised nature of most academic environments limiting the sources of information and an implicit assumption that information and knowledge are synonymous. These factors have led to overemphasis on learner modelling in the traditional ITS research, which seeks to enhance the interaction between the ITS as the provider and the learner as the consumer of knowledge, ignoring the crucial role played by the teacher in enhancing the learning in a given context. The limitations of the traditional approach become more visible when educational information is sought to be transmitted across long distances and the need for adaptation to local contexts becomes apparent. This paper argues that the human teacher, as the manager of learning, plays a vital role within the joint cognitive system consisting of the teacher, ITS, learner and learning peers. This role needs to be recognised by ITS designers by through a teacher model. It also suggests that ITS may perhaps best embody the emerging framework of Informing Science.
\end{abstract}

Keywords: Intelligent tutoring systems, Environmental contexts, Teacher model, Informing Science.

\section{Introduction}

Although computers are increasingly being used at all levels within the curriculum, their use has mainly been as work tools for data and word processing or for preparing charts and presentations or for the purpose of communication in the form of discussion boards or email. They also provide a convenient, flexible and searchable storage for various kinds of information as well as a repository for the learner's own work. Though quite important, these are ancillary uses to support the learning activities. Their use for tutoring has been quite limited in spite of years of research in Intelligent Tutoring Systems (ITS), Expert Systems and Hypertext. The more successful tutoring systems have been based around simulation for developing physical skills and procedural systems for cognitive apprenticeship

Material published as part of this proceedings, either on-line or in print, is copyrighted by the author with permission granted to the publisher of Informing Science for this printing. Permission to make digital or paper copy of part or all of these works for personal or classroom use is granted without fee provided that the copies are not made or distributed for profit or commercial advantage AND that copies 1) bear this notice in full and 2) give the full citation on the first page. It is permissible to abstract these works so long as credit is given. To copy in all other cases or to republish or to post on a server or to redistribute to lists requires specific permission from the author. based learning. The researchers in the field are now hoping that the new avenues of Multimedia, Hypermedia and Internet will somehow resolve the lack of real progress. Too much of the research is being driven by technical possibilities and the fascination with programming (Cumming, 1993) and it appears that inadequate attention is paid to the area of application, resulting in the over-ambitious projects failing to deliver anything substantial (Major et. al., 1992). It also appears that the user modelling aspect has received more than its fair share of attention at the expense of adequate consideration of the fundamental educational issues such as what constitutes knowledge, what are the methods of knowledge acquisition for these constituents, how suitable are current technologies for creation of knowledge in these constituents and how to test, using computers, the level of acquired knowledge (Cumming, 1993).

The attention received by the learner modelling, a notion borrowed from the user modelling aspect of the Human Computer Interaction (HCI) branch of Computer Science, has failed to address the diversity and richness of the educational environment. In terms of the typical user attributes - nursery to post-graduate schools, post adult education, 


\section{Patel, Kinshuk, Russell and Oppermann}

distance education and diverse subject disciplines are indicative of the scope and size of the research problem, let alone identifying and catering for individual learning style differences. Any theories that can saddle such a vast scope are likely to be too abstract to be practically useful in developing concrete applications. The experience so far has proved that the issues relating to the context of ITS cannot be wished away as they determine the type and scope of tutoring systems that are likely to succeed - especially as technologies such as natural language processing and artificial intelligence are still too basic to even attempt to mimic a human teacher based tutoring system in its entirety.

The disenchantment with the Expert Systems has given way to the expectations that the neural networks architecture may make it possible to design useful systems without really understanding the chains of causal relationships, and this may indeed happen, but as yet there are no major applications to support the belief in any substantial way. With the increasing sophistication in technology, further depth and new areas will be covered by the ever-increasing span of the educational technologies, but the increasing span by itself will not translate into the increasing scope of their use. It appears that more attention to the basic educational issues is essential to produce any substantial and usable tutoring system.

This paper is based on such consideration and the experience of developing and implementing cognitive apprenticeship based applications developed by the Byzantium project under the Teaching and Learning Technology Programme (TLTP) of the higher education funding councils of the United Kingdom and so far used by more than 10,000 students at various institutions of Higher Education. For the description of the functionality of these applications please see Patel, Kinshuk and Russell (2000).

\section{The Contexts of an ITS}

The need for considering other contexts, beyond the interaction contexts hitherto considered by ITS designers, emerged from various inter-disciplinary deliberations undertaken during the design, development and implementation of the Byzantium applications. It has also benefited from the on-going discussion on proposed further developments including implementing the methodology on the World Wide Web with a view to share both the development activities and their outcomes (see Patel, Kinshuk \& Russell, 1999).
The interaction contexts are needed in an intelligent system to help accommodate the notions of co-operation, explanation and incremental knowledge acquisition. The literature points to the notion of context being employed primarily with respect to the tasks of plan recognition, knowledge structuring, knowledge representation, reasoning, and discourse management. Obviously consideration of the context facilitates intelligent feedback by the system. Widmer \& Kubat (1993) have described a system that implements incremental concept learning in dynamic environments where the target concepts may be contextdependent and may change drastically over time. An example of the use of context for providing better reasoning is found in Walther et. al. (1992) and its use for plan recognition has been explored by Johnson (1995). They all suggest appropriate design philosophy employing the notion of context as limited to the human-computer interaction. However, the other classes of contexts, particularly the environmental contexts, are perhaps even more important for wider use of ITS in the real learning environment.

The environmental contexts of ITS are analogous to the contexts of office application systems which can be defined bi-dimensionally in terms of user attributes and nature of the tasks, whereas those of the ITS have to be described multi-dimensionally in terms of the learner, the learning environment, the learning goals and the work environment where the knowledge will be applied in due course. These contexts may be grouped around: (i) the learner's capabilities, preferences and motivation (also including learning peers); (ii) the preferences and outlooks of the teachers designing and implementing ITS; (iii) the nature of the subject discipline; (iv) the characteristics of the domain knowledge; (v) the capabilities of the tutoring medium such as the computer hardware and software capabilities; and (vi) the socio-economic environment within which the ITS is implemented (see Patel et. al., 1998).

This paper, in the main, addresses the teacher as a very important environmental context of an ITS. While the ITS designer can use the general HCI guidelines for designing a usable interface, this in itself cannot ensure adequate motivation to use the system. The designer needs to know more about "what motivates the target users of a particular ITS?" The issue is less about accommodating the novice and expert users of a particular application and more about identifying and addressing the target user sophistication as determined by age, experience, social and economic background, prior education and possibly a lot of other 


\section{Intelligent Tutoring Systems}

factors. The educational setting is likely to become increasingly less homogenous and multi-cultural and with the Internet offering a potentially global scope for an educational system, problems arising from the heterogeneity of target population have to be addressed.

As Bourges-Waldegg \& Scrivner (1998) noted, "Designing interfaces for culturally diverse users is fundamentally a problem of communicating the intended meaning of representations ... in every culturally determined usability problem a divergence between the target meaning and the interpreted meaning of representations was present." They found, however, that intercultural communications between users are less problematic since the users develop jointly a communication space in order to succeed in their task, despite differences in culture and language. Their observations suggest three important messages for the ITS designers: (i) the selection of representations need a great care and multiple representations may be necessary; (ii) synchronous or asynchronous communications based on video conferencing, web meetings, electronic white boards or discussion forums enable the explanation and negotiation of meaning. Such communications need to be systematically integrated in the learning environments; and (iii) the implementing teacher is a source of such explanation and negotiation of meaning on behalf of the ITS designer when the learner cannot understand a representation within the ITS. The pedagogy employed by the ITS, therefore, needs to be clear and possibly in harmony with that employed by the implementing teacher.

The complex representations of knowledge often arise from implicit knowledge, implied context and inferred semantic. To encounter the ambiguities of the day-to-day language and to avoid excessive use of qualifiers, each discipline creates its own 'dialect of discourse' by adopting a commonly accepted terminology. The learner has to, therefore, gain knowledge of an appropriate language of technicality and abstraction for learning a discipline, (Daniels, 1995). Though the terminology simplifies intradisciplinary communications, the extent to which its rhetoric is inconsonant with the everyday use of language, it increases the complexity perceived by the uninitiated and novice learners.

Constructivist arguments favour co-operative learning and problem-solving groups as a means of facilitating generative learning. Some of the generative activities engaged in by co-operative group learners include (Brown et. al., 1989):

- Collective problem-solving. Groups encourage synergistic insights and solutions that would not come about individually.

- Confronting ineffective strategies and misconceptions. Teachers do not have enough time to hear what and how the learners are thinking. Groups draw out, confront, and discuss both misconceptions and ineffective strategies.

- Development of collaborative work skills. Learners work together as a team through a give-andtake interaction rather than just dividing the workload at the outset.

While there is a great merit in these arguments and cooperative learning indeed motivates and stimulates more advanced learners, its suitability is questionable at the level of novice learners of a discipline. The novice learners who may still be coming to grips with the basic concepts of the domain and may also be lacking in meta-cognitive skills are more likely to be in a confusing situation that is nicely captured in the story of ten blind men trying to figure out what is an elephant! While the natural intelligence and communication ability of human learners may allow them to co-operatively construct the knowledge from such a group situation, the process may take very long time and in the absence of adequate meta-cognitive skills there could be frequent breakdowns. Some prior instruction from the teacher is likely to provide the catalyst for speeding up this process and make more effective use of the learner's time and effort.

It is interesting to note how diversely the constructivist and instructivist approaches, in their purest form, view the learner's natural intelligence. The constructivist approach relies on the learner's natural intelligence and aims to provide situational experiences to enable the learner to construct the knowledge, individually or as a member of a group. The instructivist approach, on the other hand, views the learner's mind as a container that can be filled with knowledge transferred from the teacher. In many cases the ITS designed around the instructivist approach provides so many instructions that it conveys an assumption of complete ignorance on the learner's part. Very novice learners do not mind this initially but soon starts feeling resentful 


\section{Patel, Kinshuk, Russell and Oppermann}

and finds the approach patronising! The truth perhaps lies somewhere in the middle, possibly situated in the level of learning and the degree of learner sophistication. The designer can either spend a lot of time and effort in addressing the issue to achieve full and transparent adapativity or relatively easily provide adaptability so that the learner can switch off the detailed instruction. While the first may be challenging from the artificial intelligence point of view and hence more interesting for the designer, the second would be quite acceptable from the point of view of the learner. Some researchers have observed that the human tutors virtually never provide the sort of explicit diagnosis of learner misconceptions that is sought to be provided in the traditional ITS (Cumming, 1991).

\section{The Role of the Teacher}

The over ambitious nature of the traditional ITS research also manifests itself in the almost complete exclusion of a human teacher's role in the process of computer assisted learning. It is essential to view any tutoring system as a joint cognitive system (Dalal \& Kasper, 1994) involving the tutoring software, teacher and learner (also the learning peers). The learner-ITS interaction, where the ITS is nontrivial, extends far beyond the scope of the routine HumanComputer Interaction (HCI) studies as it is a convergence of the dynamic human psychologies of the learner and the teacher and the static cyber-psychology of an ITS - reflecting the psychology of the ITS designers including their perception of learners, teachers and the learning process in an interactive but nevertheless automated ITS.

The teacher plays various roles including those of setting the scene, providing context at an appropriate grain size, sequencing the introduction of new concepts and reinforcement of the concepts already learnt, providing alternative representations, presenting alternative perspectives, selecting and scheduling appropriate educational technologies, managing the curriculum, periodically assessing the knowledge gained and overseeing the learning progression. As discussed before, the teacher acts as an agent of the designer of any educational resource, including ITS, as the learner can only question the teacher in case of any confusion or perceived shortcoming. The teacher has to go further than providing effective information in a given instance and reflect on the whole structure of the knowledge that the curriculum is designed to create in a group of learners in a given time frame. The teacher has to worry not only about the effectiveness of communication but also about the cognitive aspects of the learner's mind and present the information in an appropriate sequence to fall in proximity to the learner's current level of knowledge to enable more efficient extension of the learner's knowledge. Since it would be very difficult if not impossible in the modern classroom setting for the teacher to extensively deal with individual learners, the teaching strategy has to ensure periodic pauses and consolidation to let the weaker members of the group to catch up. Thus a successful teacher can easily be regarded as the highest order practitioner of Information Science, Cognitive Science and Management.

The other practitioners of Information Science generally provide information to those who are knowledgeable about the subject matter. For instance, a Management Accountant can expect a Manager to ask for further specific information if that provided in the first instance is too abstract or too summarised for the Manager's purpose. Thus the consumer of information is in a power relationship with the provider of the information and is generally well motivated to receive the information. In case of the teacher a converse situation exists. It is the teacher who is in a power relationship with the learners and barring some learners the others may have to be motivated to receive the information. Within this power relationship, the preferences of a teacher may prove to be more important than the individual learning style of a learner, hitherto the mainstay of ITS designers. Identifying these preferences is a difficult task as teachers have different personality and different teaching style born out of their traditional, progressive or vocational outlooks and possibly their own learning style (Entwistel, 1981). The literature also reports that the orientation to teaching strongly influences the teaching methods adopted, learning tasks set, assessments demands made and the overall workload specified (Gow \& Kember, 1993). These, in turn, influence the learner approaches to learning.

\section{The Need for a Teacher Model}

An ITS that attempts to intervene in the complex tasks carried out by the teacher needs to be 'teacher-aware'. For effective collaboration between the teacher and ITS it is necessary that the ITS can adjust to different teaching styles, at least in a broad sense. The current practice of a group of teachers using an authoring language to create an ITS is likely to produce an ITS that is biased towards a particular teaching style if the group thinks alike or a compromise that may fail to satisfy anybody. Unless the ITS has some conception of a teacher through a teacher model and enables its configuration to suit the implementing teacher, it may not find easy acceptance. Whereas the work 


\section{Intelligent Tutoring Systems}

on learner modelling has benefited by the user modelling research in the field of $\mathrm{HCI}$, the research on the role of a teacher as an implementer in the computer integrated learning environments is almost non existent.

Till adequate research results become available to effectively inform the ITS designer, it would be worthwhile to remember that the teacher-learner interaction is a very complex phenomenon across a very large network of possible convergence of teaching and learning styles, shaped by personality, background, motivation and host of other factors. It may be more productive to design small and teacher-adaptable teaching and learning tools to enhance the teacher's reach and scope in the first instance and gradually build up the teaching technology.

\section{Conclusion}

In conclusion, it may be useful to draw parallels between the Cohen's (1999) Informing Science Framework and this paper's recommendation for the consideration of environmental contexts of ITS, particularly the teacher-model. Cohen mentioned that the first component of the Informing Science framework is the Informing Environment that can be viewed at three levels of abstraction and gave an academic example to explain these levels: (1) teaching a course someone else has designed; (2) designing a course that will be taught by others; and (3) creating a new curriculum. The second component is the Delivery System and the third is the Task-Completion System. Since ITS exhibits a degree of Intelligence, it has to collaborate within the Informing Environment and can only do so if it is 'teacher-aware' through a teacher model. Some other environmental contexts mentioned in the paper are: the nature of the subject discipline, the characteristics of the domain knowledge and the capabilities of the tutoring medium such as the computer hardware and software capabilities. These contexts map onto the Delivery System component, while the teacher's role in assessing the learner knowledge as well as the last environmental context i.e. the socio-economic environment within which the ITS is implemented, map onto the Task-Completion System component of the framework. The discussion on the ITS perhaps enables a richer picture of the framework and provides a glimpse of the issues that may need to be addressed as Information systems become more intelligent.

\section{References}

Bourges-Waldegg, P. \& Scrivener, S. A. R. (1998). Meaning, the central issue in cross-cultural HCI design. Interacting with Computers, 9, 287-309.

Brown, J. S., Collins, A. \& Duguid, P. (1989). Situated cognition and the culture of learning. Educational researcher, January-February, $32-42$.

Cohen, E. (1999). Reconceptualizing Information Systems as a Field of the Transdiscipline Informing Science: From Ugly Duckling to Swan. Journal of Computing and Information Technology, 7 (3), 213-219.

Cumming, G. (1991). Using artificial intelligence to achieve natural learning. Advance Research on Computers in Education (Eds. R. Lewis \& S. Otsuki), Elsevier, 51-62.

Cumming, G. (1993). A perspective on learning for intelligent educational systems. Journal of Computer Assisted Learning, 9 (4), 229238.

Dalal, N. P. \& Kasper, G. M. (1994). The design of joint cognitive systems: the effect of cognitive coupling on performance. International Journal of Human-Computer Studies, 40, 677-702.

Daniels, H. (1995). Pedagogic Practices, Tacit Knowledge and Discursive Discrimination: Bernstein and post-Vygotskian research. British Journal of Sociology of Education, 16 (4), 517-532.

Entwistel, N. (1981). Teaching styles, learning and curricular choice. Styles of Learning and Teaching, John Wiley \& Sons Ltd., 225242.

Gow, L. \& Kember, D. (1993). Conceptions of teaching and their relationship to learner learning. British Journal of Educational Psychology, 63, 20-33.

Johnson, W.L., (1995) Plan recognition in a situational context. The 1995 IJCAI Workshop on The Next Generation of Plan Recognition Systems, Montreal, Canada.

Major, N. \& Reichgelt, H. (1992). COCA: A shell for intelligent tutoring systems. Lecture Notes in Computer Science, 608, 523-530.

Patel, A., Russell, D., Kinshuk, Oppermann, R. \& Rashev, R. (1998). An Initial Framework of Contexts for designing Usable Intelligent Tutoring Systems, Information Services and Use, 18 (1-2), 65-76.

Patel, A., Kinshuk \& Russell, D. (1999). HyperITS: A web-based architecture for evolving a configurable Learning Environment, Staff and Educational Development International, 3 (3), 265-280.

Patel, A., Kinshuk \& Russell, D. (2000). Intelligent Tutoring Tools for cognitive skill acquisition in life long learning, Educational Technology \& Society, 3 (1), 32-40.

Walther, E., Eriksson, H. \& Musen, M. A. (1992). Plug-and-Play : Construction of task-specific expert-system shells using sharable 
Patel, Kinshuk, Russell and Oppermann

context ontologies, Proceedings of the AAAI Workshop on Knowledge Representation Aspects of Knowledge Acquisition, San Jose, CA, AAAI, 191-198.

Widmer, G. \& Kubat, M. (1993). Effective learning in dynamic environments by explicit context tracking, Lecture Notes in Artificial Intelligence, 667, 227-243.

\section{Biographies}

Ashok Patel is Director, CAL Research at De Montfort University, United Kingdom.
Kinshuk is Senior Lecturer at Massey University, New Zealand.

David Russell is Director, Postgraduate Research Program at Business School, De Montfort University, United Kingdom.

Reinhard Oppermann is Professor and Researcher at German National Research Centre of Information Technology, GMD-FIT, Germany. 